\title{
Necrosis de úvula
}

\author{
Uvula necrosis
}

\author{
Mariel Andrea Del Río Parra, ${ }^{*}$ Paola Guadalupe Villatoro Portillo, * Alejandro Vela Torres ${ }^{\ddagger}$
}

\section{Resumen}

La odinofagia es un síntoma común después de una anestesia general y no requiere de un tratamiento específico. La necrosis de úvula es una complicación rara y poco reportada en la especialidad de anestesiología. Se presenta el caso de una mujer de 31 años con síntomas compatibles con enfermedad de reflujo gastroesofágico severo diagnosticado mediante una panendoscopia. Posteriormente se programa para una funduplicatura laparoscópica bajo anestesia general. A las 48 horas presenta odinofagia severa que no cede a AINES. A la exploración se observa necrosis de úvula, manejada con tratamiento conservador por parte del Servicio de Otorrinolaringología. Aunque la odinofagia después de una intubación orotraqueal es muy común, la sensación de cuerpo extraño o síntomas persistentes nos debe hacer sospechar de una posible necrosis de úvula como diagnóstico diferencial.

Palabras clave: Odinofagia, necrosis de úvula.

\section{INTRODUCCIÓN}

La odinofagia postoperatoria es un síntoma común después de la anestesia general con una incidencia reportada de hasta $62 \%^{1,2}$ y, con frecuencia los médicos la consideran una complicación relativamente menor sin requerir tratamiento específico.

La necrosis de úvula es una complicación directamente relacionada con la intubación orotraqueal, procedimientos endoscópicos o aspiración del contenido gástrico manifestándose principalmente con odinofagia severa, ${ }^{3,4}$ la cual mejora con manejo conservador a base de esteroides, antiinflamatorios no esteroides $y$, en caso de requerirse, antibióticos. ${ }^{5,6}$

\section{Abstract}

Odynophagia is a common symptom after general anesthesia which does not require a specific treatment. Uvular necrosis is a rare complication rarely reported in anesthesiology. We present a case report of a 31 years old woman with severe symptoms of gastroesophageal reflux disease and diagnosed by endoscopy. She underwent laparoscopic fundoplication with general anesthesia. 48 hours after the procedure, she presents severe odynophagia that persists after nonsteroidal anti-inflammatory drugs (NSAID). At the physical exam we observe uvular necrosis. Treated with conservative measures by otorhinolaryngology service. Besides odynophagia after orotracheal intubation is really common, persistent symptoms or foreign body sensation should make us suspect in a uvular necrosis as a differential diagnosis.

Keywords: Odynophagia, uvular necrosis.

\section{PRESENTACIÓN DE CASO}

Mujer de 31 años de edad con antecedente de pirosis, disfagia y reflujo gastroesofágico de seis meses de evolución sin tratamiento médico. Ingresa al servicio de urgencias por haber presentado dolor tipo urente en epigastrio sin irradiaciones de 24 horas de evolución, EVA 4/10, sin asociaciones. A la exploración física: TA 106/67 mmHg, FC 75 lom, FR 19 rpm, SatO 2 97\% con $\mathrm{FiO}_{2}$ al 21\%, Temp. $36^{\circ} \mathrm{C}$. Abdomen distendido, peristalsis presente, dolor a la palpación media y profunda en epigastrio y mesogastrio, sin datos de irritación peritoneal. Resto de la exploración sin datos patológicos. Se realizan análisis de laboratorio dentro de parámetros normales. Se interconsulta a
* Residente de Anestesiología.

‡ Residente de Cirugía General.

Hospital Ángeles Metropolitano.
Correspondencia:

Mariel Andrea Del Río Parra

Correo electrónico: mariel.drp@gmail.com

Aceptado: 25-03-2020.

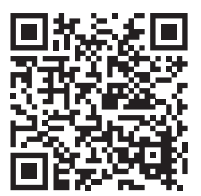


especialista de gastroenterología, tras haber realizado panendoscopia se emite el diagnóstico de enfermedad por reflujo gastroesofágico y se programa al día siguiente para realizar cirugía de funduplicatura laparoscópica. Vía aérea: úvula elongada, Mallampati I, Patil-Aldreti I, distancia interincisiva I, distancia esterno-mentoniana I, retracción mandibular I.

Se realiza procedimiento quirúrgico bajo anestesia general balanceada con inducción endovenosa. Intubación directa atraumática con hoja McCoy No. 4, visualizando Cormack-Lehane I, y tubo orotraqueal de $7 \mathrm{~mm}$ en posición lateral de la úvula con neumotaponamiento $4 \mathrm{~cm}^{3}$. Durante transoperatorio hemodinámicamente estable, concluye procedimiento quirúrgico en 70 min sin eventualidades. Se aspiran secreciones con sonda Yankauer y extuba sin complicaciones. Pasa a sala de recuperación con Aldrete de 9 , y es dada de alta con dolor de heridas quirúrgicas EVA 4/10 con AINES endovenosos.

$\mathrm{Al}$ segundo día postoperatorio refiere odinofagia severa y sensación de cuerpo extraño en faringe. A la exploración se observa úvula con resto purulento, edematosa, con tejido necrosado y mal olor a la apertura bucal (Figura 1). Sin fiebre ni linfadenopatías. Se interconsulta con especialista en ORL quien diagnostica uvulitis necrosante.

Se explica a la paciente que el problema era autolimitado y que la parte necrosada de la úvula se separaría. Se trató conservadoramente con dieta blanda, antibióticos (levofloxacino y ceftriaxona), analgésicos, e isodine bucofaríngeo. En cuatro días el tejido necrosado se resolvió sin secuelas y la clínica que presentaba la paciente desapareció.

\section{DISCUSIÓN}

El caso que se presenta se trata de paciente femenino de 31 años que después de un procedimiento quirúrgico bajo anestesia general presenta odinofagia severa que no cede a AINES, siendo diagnosticada por clínica con necrosis de úvula.

La necrosis de úvula se asocia a intubación orotraqueal y a procedimientos endoscópicos, siendo ésta una complicación con una tasa de incidencia muy baja. ${ }^{1}$ En cuanto a la etiología aún no está bien estudiada, ya que influyen diversos factores así como pocos casos reportados. ${ }^{1,2}$ Sin embargo, se piensa que la mecánica de la lesión es la compresión de la úvula ya sea por el tubo orotraqueal o el endoscopio. ${ }^{4-6}$ Asimismo, se asocia a succión orofaríngea con sonda rígida y el tamaño de la úvula. ${ }^{3}$

La característica principal de presentación es la odinofagia postoperatoria. ${ }^{1}$ En un estudio de revisión sistemática de 1,343 artículos, de los cuales únicamente se incluyeron 54, se concluye que los factores de riesgo de odinofagia en el postoperatorio son: sexo femenino, enfermedad pulmonar existente, intubación traqueal sin bloqueo neuromuscular, duración de anestesia, presencia de un tubo traqueal con restos hemáticos en la extubación, diámetro interno del tubo endotraqueal así como las altas presiones del manguito del tubo traqueal. ${ }^{7} \mathrm{De}$ éstos, nuestra paciente cuenta con la mayoría de factores de riesgo, a excepción de antecedente de enfermedad pulmonar y uso de relajante muscular.

La experiencia del anestesiólogo que realiza la intubación traqueal parece no tener influencia en la incidencia en adultos, aunque sí en niños. ${ }^{7}$ Uno de los factores más significativos es la presión excesiva del manguito, por eso el dispositivo de vía aérea supraglótica i-gelTM produce menor incidencia de dolor de garganta postoperatorio.

La literatura médica muestra casos con similar evolución y pronóstico. Un inicio precoz de la sintomatología, que evoluciona muy rápido ( $<48$ horas), se resuelve relativamente rápido sin secuelas y con manejo conservador. El tejido necrótico es bien delimitado. En esta paciente es posible que la úvula se haya plegado sobre sí misma, lo que interrumpe mecánicamente el suministro de sangre distalmente. La clara demarcación entre las porciones necróticas y sanas de la úvula que se muestran en la imagen (Figura 1), respalda esta hipótesis.

Aunque es controvertido cuál debe ser el manejo más adecuado, el tratamiento es conservador a base de esteroides, antihistamínicos y adrenalina tópica. Si no hay signos

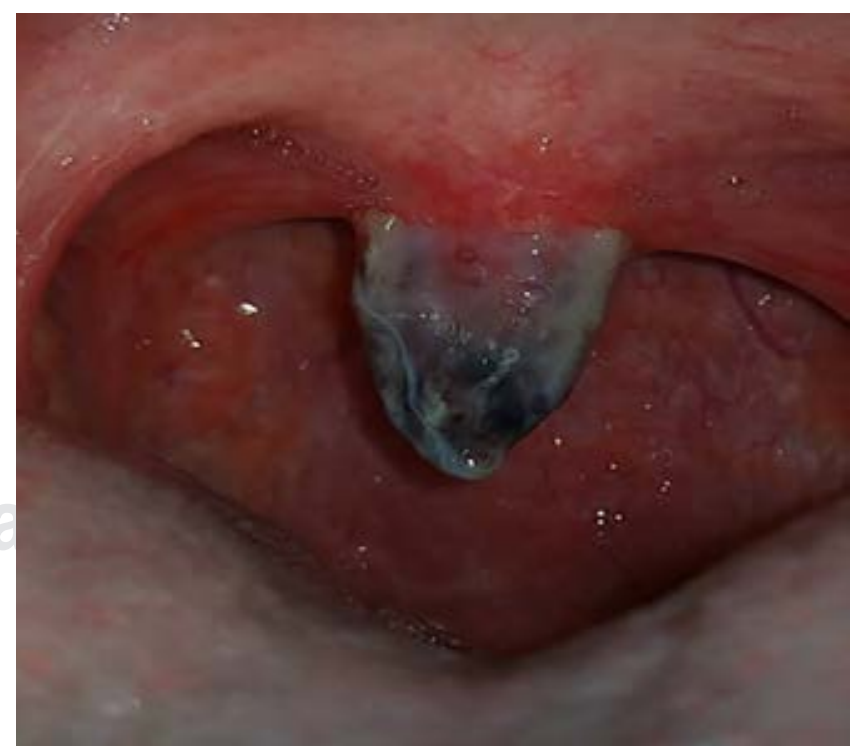

Figura 1: Úvula edematosa, purulenta y con tejido necrosado que abarca $1 / 3$ de la úvula. 
de infección, el uso de antibióticos no está inicialmente indicado.

El motivo de la presentación del caso clínico es conocer la importancia clínica de una enfermedad relativamente benigna, que puede adquirir características de severidad alarmantes si no se conoce su patogenia.

\section{CONCLUSIÓN}

Tomando en cuenta que la característica principal de esta patología es la odinofagia, es importante mantener vigilados a los pacientes en el postoperatorio por lo menos 48 horas, haciéndonos sospechar de necrosis de úvula si la odinofagia persiste después de este tiempo a pesar de tratar el dolor con analgésicos convencionales. Es una complicación autolimitada y de buen pronóstico.

\section{REFERENCIAS}

1. Evans D, Lo B. Uvular necrosis after orotracheal intubation. The Am J Emerg Med. 2009; 27 (5): 631.e3-631.e4.

2. Iftikhar M, Raziq F, Laird-Fick H. Uvular necrosis as a cause of throat discomfort after endotracheal intubation. BMJ Case Reports. 2019; 12 (7): e231227.

3. Goldin M, Ji L. Uvula necrosis, an atypical presentation of sore throat. J Emerg Med. 2013; 44 (1): 185-186.

4. Jayakumar D, Pratheema R, Ramakrishnan N. Ulcerative necrosis of the uvula following endotracheal intubation. Intensive Care Med. 2015; 41 (9): 1713-1713.

5. Sunio L, Contractor T, Chacon G. Uvular necrosis as an unusual complication of bronchoscopy via the nasal approach. Respir Care. 2011; 56 (5): 695-697.

6. Budde A, Parsons C, Eikermann M. Uvula necrosis after fibreoptic intubation. Br J Anaesth. 2018; 120 (5): 1139-1140.

7. El-Boghdadly K, Bailey CR, Wiles MD. Postoperative sore throat: a systematic review. Anaesthesia. 2016; 71: 706-717. 